\title{
TDS-OFDM based Digital Television Terrestrial Multimedia Broadcasting
}

\section{Standards}

\author{
Jian Song, Chao Zhang, \\ Jintao Wang, Yonglin Xue, \\ Changyong Pan, Kewu Peng, \\ Fang Yang, Jun Wang, \\ Hui Yang, Yu Zhang \\ and Zhixing Yang
}




\title{
TDS-OFDM based Digital Television Terrestrial Multimedia Broadcasting Standards
}

\author{
Jian Song, Chao Zhang, Jintao Wang, Yonglin Xue, Changyong Pan, Kewu Peng, Fang Yang, Jun \\ Wang, Hui Yang, Yu Zhang, and Zhixing Yang
}

\begin{abstract}
As the most popularly utilized broadcasting network, digital terrestrial television broadcasting (DTTB) can provide multimedia information coverage for the broad audience in a very efficient way because of its characteristic of wide-range coverage and mobile reception ability. After promulgating the first generation DTTB standard, digital terrestrial/television multimedia broadcasting (DTMB), in 2006, China began to research and develop the next generation DTTB standard, namely DTMB-advanced (DTMB-A), aiming to support higher spectrum efficiency and further improve transmission reliability. In 2019, DTMB-A was accepted by ITU as the second generation international DTTB standard (as System C). Similar to DTMB, time-domain synchronous - orthogonal frequency division multiplexing (TDS-OFDM) based multi-carrier modulation scheme is adopted by DTMB-A. Thanks to the more flexible frame structure, advanced error correction coding and improved constellation mapping, DTMB-A offers $30 \%$ higher transmission capacity than DTMB under the same transmission conditions. Thus, DTMB-A can support both fixed and mobile reception more efficiently, and provide users with higher quality services such as ultra-high definition television (UHDTV). This paper first gives details of key technologies at the transmitter of DTMB/DTMB-A and introduce core algorithms at the receiver. Both laboratory test and field trial results will then be provided and analyzed, especially for the application of 4K UHDTV and single frequency network (SFN).
\end{abstract}

Index Terms-digital terrestrial television broadcasting (DTTB), digital terrestrial/television broadcasting (DTMB), DTMB-advanced (DTMB-A), time-domain synchronous orthogonal frequency division multiplexing (TDS-OFDM), ultrahigh definition TV (UHDTV)

\section{INTRODUCTION}

Digital terrestrial television broadcasting (DTTB) can meet the basic demands of "information to person" in modern society with the advantages of high power and spectrum efficiency, which offers great value. Therefore, DTTB has been extensively adopted in many countries. The first generation DTTB standards, including American advanced television systems committee (ATSC) standard [1], European digital video broadcasting - terrestrial (DVB-T) standard [2], Japanese integrated service digital broadcasting - terrestrial (ISDB-T) standard [3] and Chinese digital terrestrial/ television multimedia broadcasting (DTMB) standard [4]-[6], have

This work was supported by the China National Science Foundation under Grant 61931015 facilitated the popularization of DTTB around the world. With the continuous development of multimedia information technology, a variety of new media services and high-quality audio and video services such as augmented reality (AR), virtual reality (VR), 3-dimensional TV (3DTV), ultra-high definition TV (UHDTV), etc. are emerging. Such challenges from the new broadcasting services become great driving forces to promote the evolution of DTTB standards.

New types of multimedia services always require higher transmission data rates, which means the DTTB system needs to provide higher spectrum efficiency within the same bandwidth and the requirement for the transmission reliability also increases significantly, which is beyond the capability of the first generation DTTB standards. Therefore, the study and development of the second generation DTTB standard become very important and are now in progress in many countries.

Europe began the research of the second generation DTTB standard first and promulgated DVB-T2 in 2008 [7]. DVB-T2 adopts the latest breakthrough for modulation and coding technologies at that time, providing efficient and reliable audio/video and data transmission for fixed, portable and mobile devices. The maximum data rate of DVB-T2 is about $50.1 \mathrm{Mbps}$ in $8 \mathrm{MHz}$ bandwidth using extended bandwidth mode. Under the same planning restrictions and working conditions, DVB-T2 increases the transmission capacity by over $30 \%$ compared with DVB-T.

ATSC started the preparation of the new generation DTTB standard ATSC 3.0 in 2011. ATSC opened the solicitation of technical proposals in 2013, and technical status was basically frozen in 2015. In June 2017, the physical layer transmission standard of ATSC 3.0 (A/322) [8] has been finally released. Without the backward compatibility requirement, orthogonal frequency division multiplexing (OFDM) technology combining with advanced constellation mapping and channel coding are utilized by ATSC 3.0, supporting higher spectrum efficiency and throughput. Therefore, ATSC 3.0 gives broadcasters more choices of technical solutions.

China launched the research and development of a new generation DTTB standard, DTMB - advanced (DTMB-A) [9][10], based on the key technologies of DTMB since 2008. DTMB-A retains time-domain synchronous - OFDM (TDSOFDM) as its basic transmission technology similar to DTMB.

The authors are with the Beijing National Research Center for Information Science and Technology (BNRist), Department of Electronic Engineering, Tsinghua University, Beijing 100084, China (e-mail: z_c@tsinghua.edu.cn). 


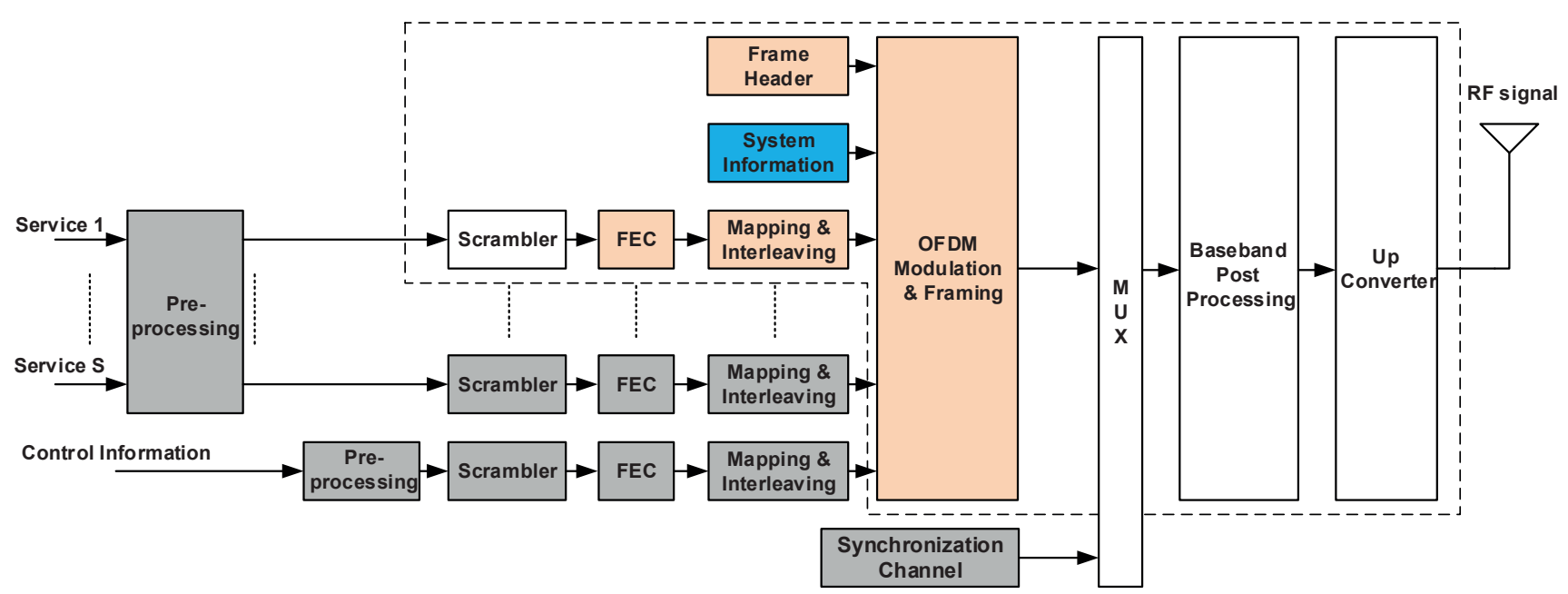

Fig. 1. Functional block diagram of the transmitter for DTMB and DTMB-A

Besides, the more flexible frame structure incorporating amplitude phase shift keying with Gray mapping (Gray-APSK) and bit-interleaved coded modulation (BICM) based on low density parity check (LDPC) code provides DTMB-A more powerful performance. Benefit from the above techniques, DTMB-A has over $30 \%$ higher spectrum efficiency than DTMB under the same transmission conditions, or in another word, possesses better immunity to interference and lower receiving threshold under the same spectrum efficiency. DTMB-A can support both fixed and mobile reception, and can support UHDTV broadcasting and high-performance single frequency network (SFN). In December 2019, DTMB-A was adopted by ITU as the System $\mathrm{C}$ of the second generation international DTTB standards with DVB-T2 as System A and ATSC 3.0 as System B [11].

Extensive laboratory tests and field trials have been conducted during the development of the DTMB-A system [12]-[14]. In 2018, the first 4K UHDTV broadcasting experimental network based on DTMB-A was established in Jiaxing City, Zhejiang Province. Moreover, in August 2019, invited by Hong Kong Television Broadcasts Limited (TVB), Tsinghua University and Beijing Digital Television National Engineering Laboratory (DTNEL) organized the field trials to evaluate the performance of SFN and UHDTV broadcasting in Hong Kong. The test results prove that DTMB-A can support reliable 4K UHDTV program transmission under the fairly complex receiving environment in Hong Kong. In August 2019, the DTMB-A based $4 \mathrm{~K}$ transmission system with $6 \mathrm{MHz}$ bandwidth was exhibited at the SET EXPO in Sao Paulo, Brazil.

Both DTMB and DTMB-A adopt TDS-OFDM as their key technology. Hence, this paper will start from TDS-OFDM, and systematically introduce the system architectures and the key techniques used by DTMB and DTMB-A. In addition, details of algorithms utilized at the receiver are also discussed as an important part of the whole DTTB system. Laboratory test and field trial results of the DTMB-A system will be then provided, especially for the application of the 4K UHDTV demonstration network.
The rest of this paper is organized as follows. Section II introduces the system architecture of the transmitter. Key technologies of transmitter and receiver are provided in Section III and Section IV respectively. Section V shows the laboratory test and field trial results. A conclusion is drawn in Section VI.

\section{SYSTEM ARCHITECTURE}

\section{A. Transmitter Structures}

In order to show the structures clearly and give a comparison between DTMB and DTMB-A system, a block diagram combining DTMB and DTMB-A transmitter modules is shown in Fig. 1. Since only the multi-carrier transmission mode of TDS-OFDM is used in DTMB-A, only the structure of the OFDM transmission is described. In Fig. 1, the parts in the dotted box constitute the DTMB transmitter, while the gray modules are extra parts for the DTMB-A transmitter. The blue module is only available in the DTMB system and the orange ones in the dotted box are common functional blocks for both systems but improved distinctly for the DTMB-A system with newly developed technologies. Thus, differences between the two systems are clear from Fig. 1.

It is illustrated that the DTMB system only supports single service broadcasting. After multiplexing the service data into standard MPEG-TS packet, scrambling, forward error correction (FEC) coding, constellation mapping and interleaving are executed successively and data constellation symbols are obtained. These symbols are multiplexed with the signaling of transmission parameters (system information) and then allocated to $C$ sub-carriers. After that, inverse fast Fourier transform (IFFT) is performed to get the time-domain signal of the frame body. Then, the frame header sequence and frame body are combined to form the signal frame. After the pulse shaping filtering and orthogonal up-conversion, the radio frequency (RF) signal is obtained and ready to be transmitted over the wireless channel.

DTMB-A has distinct improvements in comparison with DTMB. DTMB-A uses super-frame as its important 


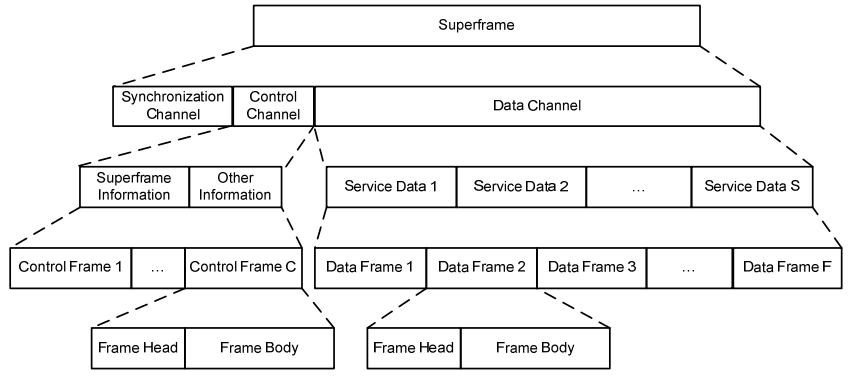

Fig. 2. Super-frame structure of DTMB-A.

transmission unit in the physical layer, and it comprises of logical channels for super-frame synchronization, control if needed, and service data. Furthermore, DTMB-A is able to support simultaneous transmission of multiple services with different quality requirements and provide independent subchannels for each of them, which fundamentally distinguishes from single service transmission mode by program multiplexing.

DTMB-A has a flexible design of frame structure based on TDS-OFDM similar to DTMB, but it pads the frame header with multi-carrier pseudo-random noise (PN-MC) sequence instead of time-domain PN sequence. It can help the receiver perform channel estimation and equalization with lower complexity and higher accuracy. Moreover, in order to adapt to different application scenarios, DTMB-A provides various optional frame specifications. The frame header supports three options including 512, 1024 and 2048 symbols, while the frame body length can be 4096 (4K), 8192 (8K) and 32768 (32K) symbols.

Amplitude phase shift keying with Gray mapping (GrayAPSK) is utilized in DTMB-A. Significant shaping gain is attained compared to traditional quadrature amplitude modulation (QAM), which enables reliable information recovery at a lower demodulation threshold. With that, DTMBA can support high-order constellation up to 256, which provides the maximum payload data rate of $49.57 \mathrm{Mbps}$ in regular bandwidth mode, or $51.00 \mathrm{Mbps}$ in extended mode. It means that under $8 \mathrm{MHz}$ RF channel and adopting the newest H.265 video compression coding, the DTMB-A system can transmit 10 HDTV programs simultaneously or one $4 \mathrm{~K}$ UHDTV program.

DTMB-A uses improved LDPC coding. The compatibility of multiple code rates and multiple code lengths is fully considered in the design. Three different code rates, including $1 / 2,2 / 3$ and $5 / 6$, and two different code lengths, including 15360 and 61440 , have been provided. At the receiver, a highthroughput parallel iterative decoder can be implemented to improve the decoding performance at the same time as keeping low hardware complexity. Additionally, LDPC decoding and constellation demapping can be combined and processed iteratively, which further lowers the threshold compared with conventional independent decoding and demapping.

DTMB-A also supports transmit diversity with two antennas as an option, which improves the robustness under deep fading channel. The diversity mode can also be applied in SFN. By applying diversity codes from different transmitters, the influence of the artificial multipath in traditional SFN can be effectively eliminated, which improves the coverage of the SFN.

\section{B. Frame Structure}

DTMB standard has a hierarchical super-frame structure, including signal frame, super-frame, minute frame and day frame from bottom to top. The signal structure has periodicity and is kept synchronized with nature time. As the basic transmission unit of DTMB, the signal frame consists of frame header and frame body, where the frame header is constituted by time-domain PN sequence and the frame body is dependent on the number of sub-carriers.

In DTMB-A, the more flexible physical layer super-frame structure is utilized which is depicted in Fig. 2. As the fundamental unit transmitted in the physical layer, the superframe is made up of synchronization channel, control channel and data channel. The synchronization channel is used for primary synchronization of the super-frame and transmitting basic parameters. $S$ services are allocated on the data channel, each of them occupies an integral number of data frames. Every super-frame has $F$ data frames, where $F$ is determined by transmission parameters. Control channel has $\mathrm{C}$ control frames, which carry the service configuration information, parameters for demapping and decoding, and fast real-time information (e.g., short message). Data frame and control frame have the same frame structure consisted of frame header and frame body, and both adopt TDS-OFDM.

The preamble of each super-frame is the synchronization channel, which is used for fast signal capture, coarse timing, estimation of carrier frequency offset, and basic transmission parameters acquisition.

Control frame is generated by using low-rate of FEC coding, quadrature phase shift keying (QPSK) mapping, symbol interleaving, IFFT, and frame header insertion. Control channel transmits the information of frame structure, service composition and other necessary information for multiple service broadcasting. Each super-frame can contain $\mathrm{C}$ control frames, which are placed right after the synchronization channel one by one. For single service mode, the control frame isn't needed and can be eliminated.

Each data frame within the data channel may have independent mode of coding and modulation, which can be configured flexibly according to the application. Similar processes in control channel are carried out to obtain data frames. Moreover, both the control frame and the data frame can have different lengths of either frame header or frame body.

Synchronization channel, data frames of all the services and the control frames are combined to compose a super-frame. Baseband post-processing is implemented to get the baseband transmission signal, which is followed by orthogonal upconversion and the RF signal is obtained.

\section{System Parameters}

Table I lists some basic transmission parameters of DTMB and DTMB-A systems. It is shown that DTMB-A has made 


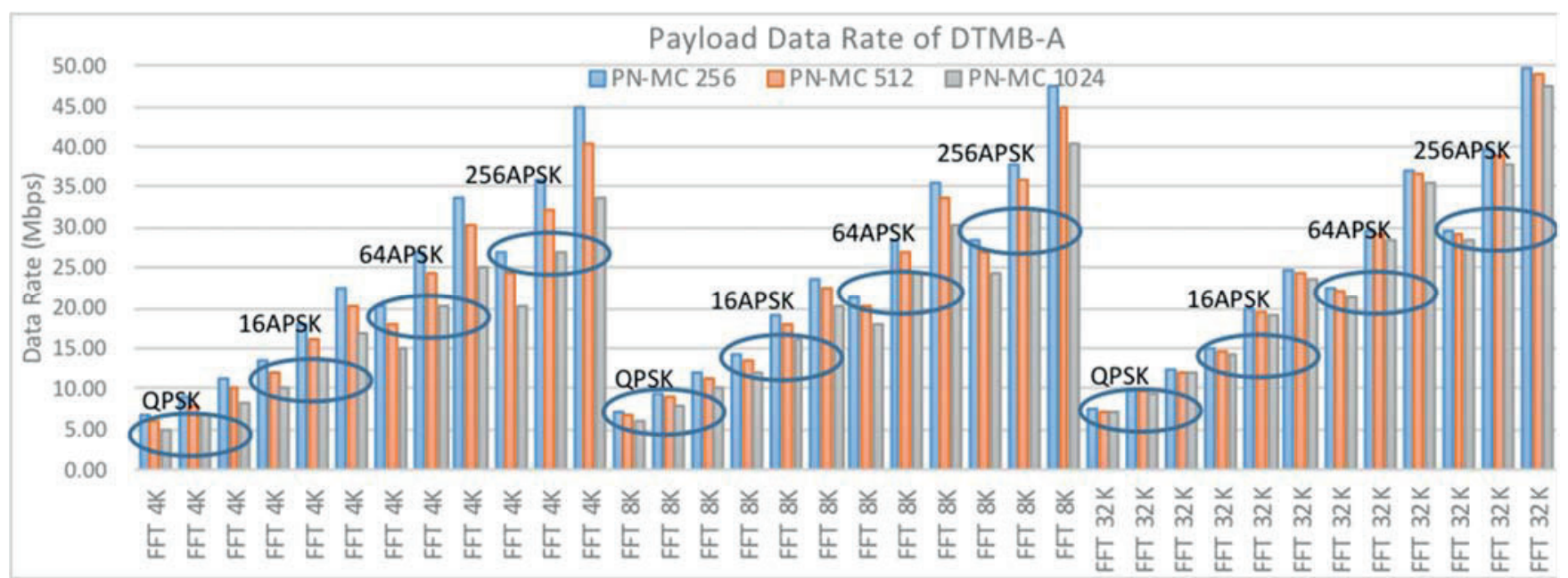

Fig. 3. Payload data rate of DTMB-A in 7.56MHz bandwidth mode.

TABLE I

TRANSMISSION PARAMETERS OF DTMB AND DTMB-A

\begin{tabular}{lll}
\hline \hline \multicolumn{1}{c}{ Parameter } & \multicolumn{1}{c}{ DTMB } & \multicolumn{1}{c}{ DTMB-A } \\
\hline Modulation & $\begin{array}{l}\text { TDS-OFDM, Single- } \\
\text { carrier }\end{array}$ & TDS-OFDM \\
FEC & BCH, LDPC & BCH (option), LDPC \\
LDPC code length & 7493 & 15360,61440 \\
LDPC code rate & $0.4,0.6,0.8$ & $1 / 2,2 / 3,5 / 6$ \\
Constellation & QPSK, QPSK-NR, & QPSK, 16APSK, \\
& 16QAM, 32QAM, & 64APSK, 256APSK \\
& 64QAM & \\
Guard interval & PN420, PN595, PN945 & 2PN-MC 256, 2PN-MC \\
& & 512,2 PN-MC 1024 \\
FFT size & 3780 & $4096,8192,32768$ \\
Roll-off factor & 0.05 & $0.05,0.025$ \\
Bandwidth & $7.56 \mathrm{MHz}$ & $7.56 \mathrm{MHz}, 70 / 9 \mathrm{MHz}$ \\
Payload data rate & $4.813 \sim 32.486 \mathrm{Mbps}$ & $5.00 \sim 51.00 \mathrm{Mbps}$ \\
Multi-service & No & Yes \\
Transmit diversity & No & Yes \\
Enhanced SFN & No & Yes \\
PAPR reduction & No & Yes \\
\hline \hline
\end{tabular}

certain enhancement on channel coding and modulation, frame structure, therefore, has higher spectrum efficiency, and can support more functions like multiple service broadcasting, enhanced SFN and so on, which makes DTMB-A satisfy the requirements of new types of broadcasting services well.

\section{Payload Data Rates}

Both DTMB and DTMB-A provide a number of options of parameters to deal with different applications. The payload data rate is determined by the choice of the parameter set, which includes frame header and frame body lengths, constellation order, channel coding rate, system bandwidth etc. Taking DTMB-A as an example, the payload data rates under different working modes in $7.56 \mathrm{MHz}$ bandwidth channel are shown in Fig. 3.

Data in Fig. 3 are divided into three groups with the respect to the FFT length of $4 \mathrm{~K}, 8 \mathrm{~K}$ and $32 \mathrm{~K}$ for the horizontal axis. Every group is further divided into four sub-groups by the constellation order. Data in the sub-groups with the same color

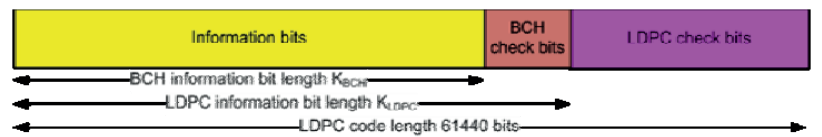

(a)

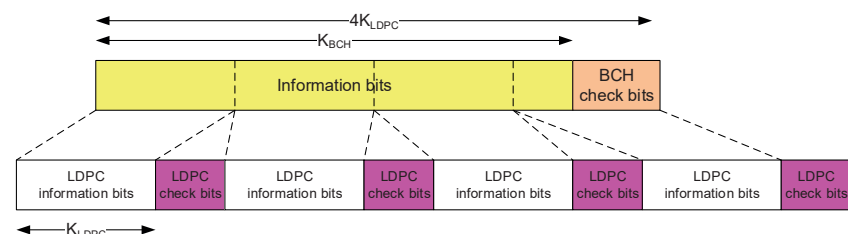

-LDPC code 15360 bits

(b)

Fig. 4. The codeword structure of the LDPC code. (a) LDPC code of 61440 bits. (b) LDPC code of 15360 bits

are arranged from left to right according to the coding rate of $1 / 2,2 / 3$ and $5 / 6$ respectively, while different colors represent different frame header lengths. Fig. 3 indicates that DTMB-A can reach the maximum data rate with PN-MC 256, 32K FFT, 256APSK and 5/6 code rate, and has the minimum data rate with PN-MC 1024, 4K FFT, QPSK and 1/2 code rate. Under the fixed frame header length and FEC code rate, the working mode with longer FFT length and higher constellation order is more suitable for the fixed reception, while mobile applications prefer shorter FFT length and lower constellation order.

\section{Bit INTERLEAVEd CODED Modulation}

\section{A. Forward Error Correction Code}

After randomization, the data stream will be encoded by FEC coding to enhance the transmission robustness.

DTMB-A uses concatenated FEC coding as DTMB, where the inner code is LDPC code and the optional outer code is $\mathrm{BCH}$ code. Using $\mathrm{BCH}$ code as the outer code can provide stronger error correction ability at a slightly lower data rate.

Comparing with Turbo code, LDPC code has lower decoding complexity [15]. By further optimizing the existing LDPC code in DTMB standard, DTMB-A uses quasi-cyclic LDPC (QC- 
TABLE II

PARAMETERS OF LDPC CODE

\begin{tabular}{c||c|c}
\hline \hline \multirow{2}{*}{ Code Rate } & \multicolumn{2}{|c}{ Information Bit Length } \\
\cline { 2 - 3 } & Code Length 61440 & Code Length 15360 \\
\hline $1 / 2$ & 30720 & 7680 \\
\hline $2 / 3$ & 40960 & 10240 \\
\hline $5 / 6$ & 51200 & 12800 \\
\hline \hline
\end{tabular}

TABLE III

PARAMETERS OF QC-LDPC CODE

\begin{tabular}{c|c|c||c|c}
\hline \hline \multirow{2}{*}{ Code Rate } & \multicolumn{2}{|c||}{ Code Length $15360, \mathrm{~b}=128$} & \multicolumn{2}{c}{ Code Length $61440, \mathrm{~b}=512$} \\
\cline { 2 - 5 } & $n$ & $k$ & $n$ & $k$ \\
\hline $1 / 2$ & 120 & 60 & 120 & 60 \\
\hline $2 / 3$ & 120 & 80 & 120 & 80 \\
\hline $5 / 6$ & 120 & 100 & 120 & 100 \\
\hline \hline
\end{tabular}

LDPC) code, which further lowers both the carrier-to-noise ratio $(\mathrm{C} / \mathrm{N})$ threshold and implementation complexity, and is also suitable for the multiple code rates and code lengths, aiming at supporting fixed and mobile reception [16]. Considering the structure of the QC-LDPC encoder, receiver performance, storage occupation, and power consumption, the optimal time-frequency interleaving scheme is applied to DTMB-A for better performance.

DTMB-A has two code length options of 15360 and 61440 and three code rate options of $1 / 2,2 / 3$ and $5 / 6$, which are shown in Table II. The code word structures of two code lengths are shown in Fig. 4 (a) and (b) respectively.

QC-LDPC code is an important subset of LDPC code and its parity check matrix has a quasi-cyclic structure. This kind of LDPC code is simple in structure, easy to design and has excellent performance. Because of the regular structure of the parity check matrix, the QC-LDPC encoder can be implemented by a relatively simple circuit while parallelization can be adopted in decoding to lower the hardware complexity. The generation matrix of the QC-LDPC code with systematic structure used by DTMB-A can be expressed as

$$
\mathbf{G}_{\mathrm{qc}}=\left[\begin{array}{ccccccc}
\mathbf{I} & \mathbf{O} & \cdots & \mathbf{O} & \mathbf{G}_{0,0} & \cdots & \mathbf{G}_{0, n-k-1} \\
\mathbf{O} & \mathbf{I} & \cdots & \mathbf{O} & \mathbf{G}_{1,0} & \cdots & \mathbf{G}_{1, n-k-1} \\
\vdots & \vdots & \ddots & \vdots & \vdots & \mathbf{G}_{i, j} & \vdots \\
\mathbf{O} & \mathbf{O} & \cdots & \mathbf{I} & \mathbf{G}_{k-1,0} & \cdots & \mathbf{G}_{k-1, n-k-1}
\end{array}\right]
$$

where $\mathbf{I}$ is $b \times b$ identity matrix, $\mathbf{O}$ is $b \times b$ zero matrix, $G_{i, j}$ is $b \times b$ circulant matrix with $0 \leq i \leq k-1,0 \leq j \leq c-1$. The values of $n, k$, and $b$ under different coding parameters are shown in Table III.

\section{B. Constellation Mapping}

DTMB standard adopts traditional QAM constellation mapping with maximum order of 64 .

Information theory suggests that only Gaussian-distributed input can achieve the channel capacity over power-limited additive white Gaussian noise (AWGN) or fading channel. Restricted by constellation, traditional regular QAM mapping won't generate the signal with Gaussian distribution, so there is a gap between theoretical channel capacity and reliable data rate under the constellation constraint. Accordingly, the technique that makes mapping output closer to the Gaussian distribution is called shaping, and the resulting gain is called shaping gain.

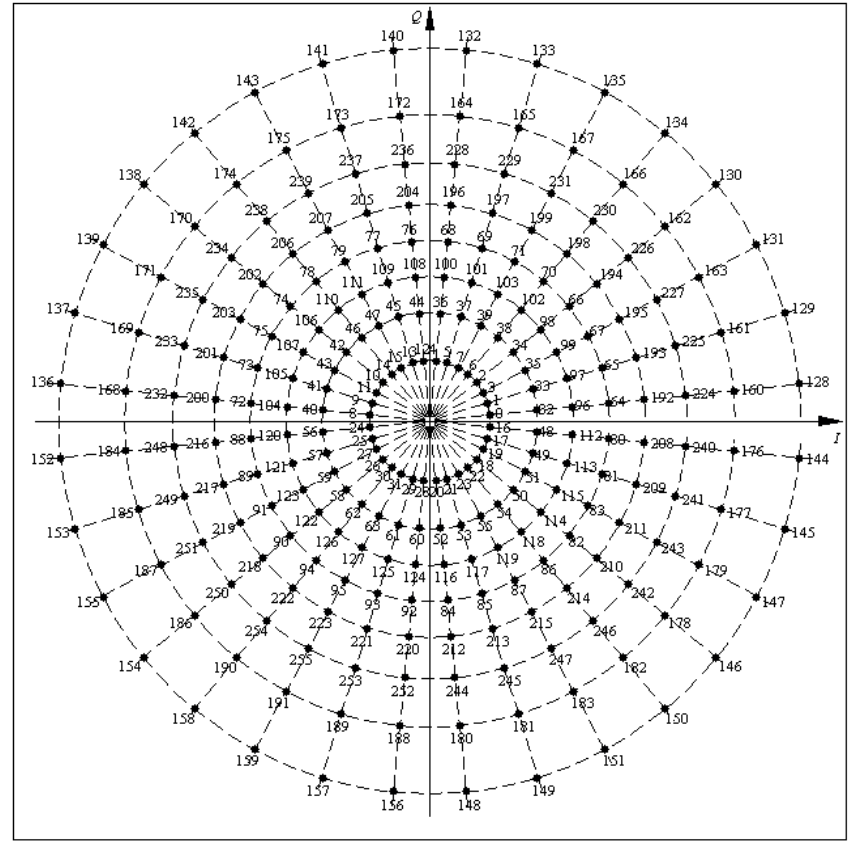

Fig. 5. Gray-APSK constellation of 256 points.

APSK constellation has a typical non-uniform distribution characteristic, which gives it notable shaping gain compared with regular QAM mapping. Gray-APSK design based on mutual information optimization theory can significantly improve channel capacity under constellation constraint and obtain shaping gain with both independent and iterative demapping [17].

Four different constellation orders are defined in DTMB-A, including QPSK, 16APSK, 64APSK, and 256APSK, in which 256APSK can support higher transmission rate and be suitable for fixed reception with high spectrum efficiency, while QPSK can provide higher robustness and be suitable for severe transmission conditions such as high-speed mobile reception. The typical constellation of 256 Gray-APSK used in DTMB-A is shown in Fig. 5.

\section{BICM-ID}

It is proved that interleaving in bit-level can bring higher diversity order to improve the performance. Thus, combining the channel coding and constellation mapping by bitinterleaving can provide better performance in fading channel. Such technique is called bit-interleaved coded modulation (BICM). If at the receiving side, the demapping process of BICM doesn't use the constraint introduced by channel coding among bits, performance loss, especially with high order constellation mapping will occur. Therefore, the output of decoding can be fed back to the demapping module to assist demapping, which forms the process of BICM with iterative decoding/demapping (BICM-ID) [18][19]. The block diagrams of both BICM and BICM-ID systems are shown in Fig. 6 (a) and (b) respectively.

Gray-APSK adopted in DTMB-A means the amplitude and phase are independent at each constellation point and both of 


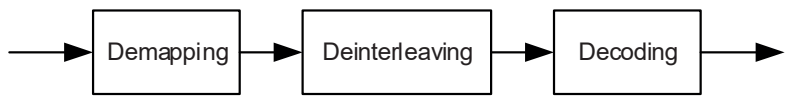

(a)

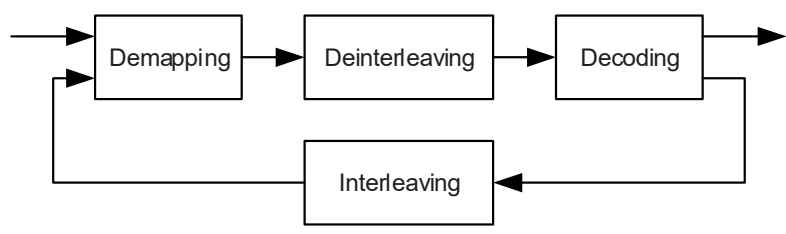

(b)

Fig. 6. Block diagram of the demapping and decoding. (a) BICM. (b) BICMID.

\begin{tabular}{|l|l|}
\hline Frame header & Frame body \\
\hline
\end{tabular}

Fig. 7. The signal frame structure of TDS-OFDM.

them are Gray mapped respectively. Thus, DTMB-A is more suitable for both independent demapping and LDPC or Turbo code based BICM-ID, which further enhances the iterative gain.

\section{TDS-OFDM BASED FRAME STRUCTURE}

\section{A. TDS-OFDM Technology}

One of the biggest challenges of terrestrial broadcast wireless transmission is the frequency selective fading caused by multipath. OFDM has natural advantages in combating it. However, a strict requirement of synchronization is needed to ensure the orthogonality among subcarriers when applying OFDM.

TDS-OFDM is adopted both in DTMB and DTMB-A as the fundamental transmission technique. TDS-OFDM is a multicarrier transmission scheme that can flexibly support time and frequency domain processing when needed. Through the combined processing of time and frequency domains, it can easily realize fast signal acquisition and robust synchronization, and also supports time-frequency two-dimensional segment for the physical layer resource allocation. The signal frame structure of TDS-OFDM illustrated in Fig. 7 consists of frame header and frame body. The frame header is a known training sequence, usually a PN sequence. It is used as the guard interval between two adjacent OFDM data blocks instead of traditional cyclic-prefix or zero-padding. Since the sequence is known by the receiver, the frame header is also used for parameter synchronization and channel estimation. Thus, TDS-OFDM does not need to insert any frequency-domain pilot which improves the spectrum efficiency obviously.

\section{B. Signal Frame Structure Based on PN-MC}

DTTB system usually works under complex multipath conditions. For example, the receiver will receive the many reflected signal from buildings and complex terrain. When SFN is applied, artificial multipath also exists and the receiver will receive the transmitted signals from several nearby transmitters

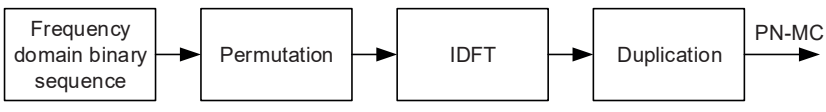

(a)

\begin{tabular}{|l|l|l|}
\hline PN-MC & PN-MC & Frame body \\
\hline
\end{tabular}

(b)

Fig. 8. PN-MC structure. (a) Generation method of PN-MC. (b) TDS-OFDM signal frame structure using dual $\mathrm{PN}-\mathrm{MC}$ as the frame header.

through different paths and processes them as echoes. The multipath signal will significantly influence the performance of the receiver, leading to a higher bit error rate and even failure of reception.

In TDS-OFDM system, the frame header can be used to facilitate fast synchronization, efficient channel estimation and equalization. Moreover, since the frame header adopts known training sequences, the accuracy of the parameter estimation can be improved remarkably under low SNR conditions. The longer the frame header is, the better it is to resist long delay echo signal, at the cost of lower payload data rate. In addition, the long frame header mode can be a preferable alternative for wide range $\mathrm{SFN}$.

DTMB system has three different frame header lengths, including PN420, PN595 and PN945. These three kinds of frame headers are based on the time-domain PN sequence, and generated by cyclic expansion (PN420, PN945) or truncation (PN595). However, in multipath channel, there is intrinsic interference between the frame header and the frame body when TDS-OFDM is used. Iterative interference cancellation algorithm is necessary to obtain high precision channel estimation, which increases the hardware complexity of the receiver.

In order to further improve the precision and also reduce the implementation complexity, DTMB-A adopts an improved frame header design method, using two repeated PN-MC sequences as the frame header. The details of the generation procedure and the frame structure are depicted in Fig. 8 (a) and (b) respectively. As illustrated in Fig. 8 (a), the known frequency-domain binary sequence is firstly interleaved in the frequency-domain by permutation operation. Then, IFFT is performed and the time-domain PN-MC sequence is obtained. Finally, the PN-MC sequence is repeated and put in front of the frame body as the frame header, creating the frame structure shown in Fig. 8 (b). DTMB-A supports three PN-MC sequence lengths of 256,512 and 1024 , as well as three frame body lengths of 4096, 8192 and 32768.

Since the frame header of DTMB-A contains two identical $\mathrm{PN}-\mathrm{MC}$ sequences, the first one can be regarded as the cyclic prefix of the second PN-MC, which effectively avoids the interference from the frame body of the former signal under multipath channels. Therefore, precise channel estimation can be obtained at slightly lower spectrum efficiency by using the second PN-MC sequence without any interference, and the iterative interference cancellation can be eliminated for the receiver compared with traditional TDS-OFDM system 


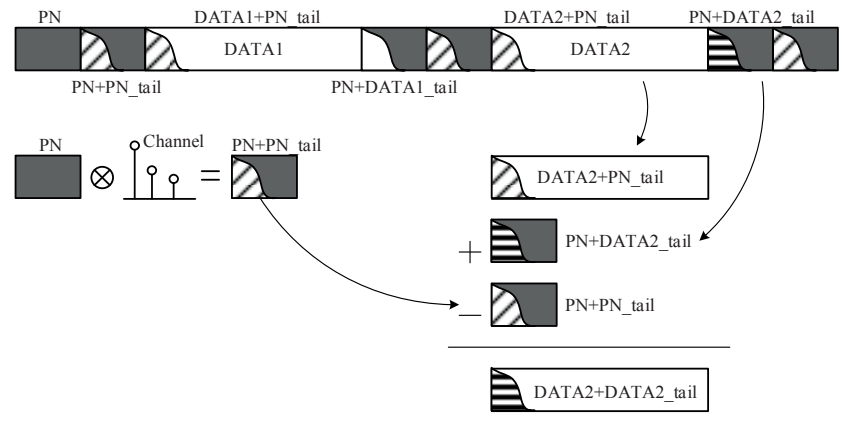

Fig. 9. The algorithm of cyclic reconstruction used in DTMB-A.

[20][21]. After the accurate channel estimating, the receiver can utilize a simple overlap-and-add algorithm to complete the cyclic reconstruction of the OFDM data block. The schematic diagram of the algorithm is shown in Fig. 9.

\section{Synchronization Channel}

The super-frame synchronization channel is a novel design in the DTMB-A standard [22]. It is placed at the beginning of each super-frame and has a special structure in both time and frequency domains. It can provide fast and robust signal detection, coarse timing and carrier estimation in low SNR conditions. At the same time, it can support fundamental signaling transmission, which helps the receiver get basic parameters of the physical layer signal and guide the subsequent demodulation and decoding.

The structure of the super-frame synchronization channel is displayed in Fig. 10. The basic unit is an OFDM symbol with the length of 1024 samples. In the frequency domain, the OFDM symbol has two identical PN sequences, namely PN_H, whose length is 256, while other subcarriers in the symbol are padded with 0 . There are $\Delta L$ subcarriers between these two PN_H sequences where $\Delta L \in[64,319]$. Therefore, it means that $\Delta L$ has 256 options which can be used to transmit 8-bit signaling information. Let $\left\{Z_{m}\right\}_{m=0}^{1023}$ denotes the 1024-point frequency-domain signal, and it can be expressed as

$Z_{m}= \begin{cases}0 & 0 \leq m<256-\lceil\Delta L / 2\rceil \\ \mathrm{PN} \_\mathrm{H}_{m-256+\lceil\Delta L / 2\rceil} & 256-\lceil\Delta L / 2\rceil \leq m<512-\lceil\Delta L / 2\rceil \\ 0 & 512-\lceil\Delta L / 2\rceil \leq m<512+\lfloor\Delta L / 2\rfloor \\ \mathrm{PN} \_\mathrm{H}_{m-512-\lfloor\Delta / 2\rfloor} & 512+\lfloor\Delta L / 2\rfloor \leq m<768+\lfloor\Delta L / 2\rfloor \\ 0 & 768+\lfloor\Delta L / 2\rfloor \leq m<1024\end{cases}$

where $\lceil\cdot\rceil$ denotes round towards plus infinity, and $\lfloor\cdot\rfloor$ presents denotes round towards minus infinity.

This kind of signaling transmission method based on distance variation is one of the important features of DTMB-A.

The frequency-domain OFDM symbol is converted to the time domain signal of length 1024 by IFFT. After that, the time domain signal is divided into two parts of $\mathrm{A}$ and $\mathrm{B}$ with the same length. Then, part B and the minus version of B are copied to the front and the end of the time domain OFDM symbol respectively as the pre and postambles, which yields the superframe synchronization channel with the special structure of $[\mathrm{B}, \mathrm{A}, \mathrm{B},-\mathrm{B}]$ in the time domain.

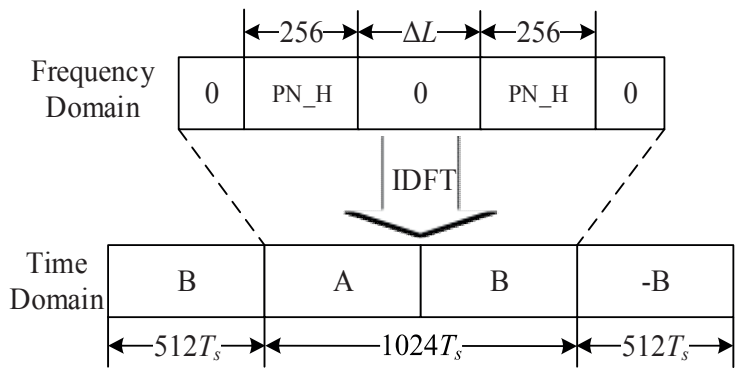

Fig. 10. The time and frequency domain structure of the synchronization channel.

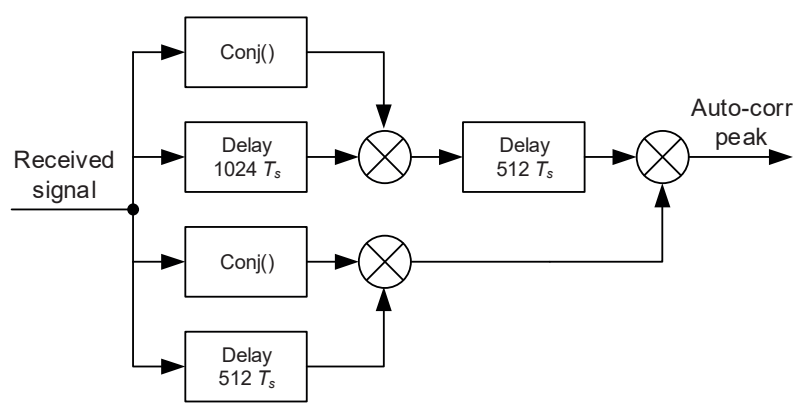

Fig. 11. The algorithm of signal detection using the synchronization channel.

One of the important functions of the super-frame synchronization channel is to realize the detection of DTMB-A signal from the received signal without any prior information, and extract the basic transmission parameter signaling for demodulation and decoding. Since there are three repeated segments of B (including an inverse one) in the time domain, the low complexity time-domain auto-correlation can be used for the signal detection. Fig. 11 gives an illustration of the algorithm of the signal detection process using the super-frame synchronization channel. The correlation between the first and the third parts of $\mathrm{B}$, and between the third and the fourth parts of $\mathrm{B}$, are calculated respectively. These two correlation results are combined by multiplication or addition to ensure a more sharp auto-correlation peak [23].

The super-frame synchronous channel can realize signal detection and recognition under the noisy environment, and ensures the fast detection of the DTMB-A signal.

\section{Field Trial Results}

The prototype verification system and commercial set-top box of DTMB-A have been fully tested in the laboratory, and field trials have been carried out in many cities and districts in China, including Changsha, Kunming, Urumchi, Jiaxing and Hong Kong. The reception performance of the DTMB-A system in various typical urban environments has been evaluated sufficiently. In this section, we will focus on the test results of $4 \mathrm{~K}$ in Jiaxing and SFN plus $4 \mathrm{~K}$ in Hong Kong, respectively. 
TABLE IV

PARAMETERS OF THE FIELD TRIALS IN JIAXING CITY

\begin{tabular}{ll}
\hline \multicolumn{1}{c}{ Parameter } & \multicolumn{1}{c}{ Value } \\
\hline Transmission system & DTMB-A \\
Bandwidth & 7.56MHz \\
Constellation & $256 \mathrm{APSK}$ \\
FFT size & $32 \mathrm{~K}$ \\
Guard interval & Dual PN-MC 256 \\
FEC & LDPC 61440 \\
FEC rate & $2 / 3$ \\
Payload data rate & $39.7 \mathrm{Mbps}$ \\
Center frequency & $562 \mathrm{MHz}$ \\
Transmission power & $1000 \mathrm{~W}$ \\
Transmission antenna & Horizontal polarized \\
Video coding & H.265 \\
Audio coding & MPEG-4 AAC \\
Transmission antenna height & $145 \mathrm{~m}$ above ground level \\
Receiving antenna height & $10 \mathrm{~m}$ above ground level \\
\hline \hline
\end{tabular}

TABLE V

TEST RESULTS IN JIAXING CITY

\begin{tabular}{|c|c|c||c|c|c|}
\hline \hline \multicolumn{3}{|c||}{ Outdoor reception } & \multicolumn{3}{|c|}{ Indoor reception } \\
\hline No. & $\begin{array}{c}\text { Signal } \\
\text { strength } \\
(\mathrm{dBm})\end{array}$ & $\begin{array}{c}\text { Margin } \\
(\mathrm{dB})\end{array}$ & No. & $\begin{array}{c}\text { Signal } \\
\text { strength } \\
(\mathrm{dBm})\end{array}$ & $\begin{array}{c}\text { Margin } \\
(\mathrm{dB})\end{array}$ \\
\hline 1 & -52.9 & 28 & 1 & -50.6 & 32 \\
2 & -65.5 & 13 & 2 & -57.9 & 18 \\
3 & -65.5 & 10 & 3 & -36 & 46 \\
4 & -72.9 & 2 & 4 & -55.6 & 27 \\
5 & -68.4 & 10 & 5 & -40.8 & 37 \\
6 & -65.2 & 11 & 6 & -39 & 40 \\
7 & -66.6 & 14 & 7 & -35.2 & 48 \\
8 & -50.2 & 32 & & & \\
9 & -61.8 & 14 & & & \\
10 & -70.2 & 4 & & & \\
\hline \hline
\end{tabular}

\section{A. $4 K$ UHDTV Broadcasting in Jiaxing City}

In order to validate the feasibility and reliability of DTMBA system for $4 \mathrm{~K}$ UHDTV program broadcasting, the experimental DTMB-A network was set up in Jiaxing City, Zhejiang Province in August 2018 [14]. Reception performance is tested at several typical sites in Jiaxing City, including both indoor and outdoor receptions. The transmission mode and the transmitter parameters are summarized in Table IV and the situations of the test sites are shown in Fig. 12.

During the measurements, 10 typical fixed outdoor and 7 representative fixed indoor receiving points were chosen, with the locations of the outdoor and indoor testing points and the corresponding transmitting stations shown in Fig. 13 (a) and (b) respectively. The received signal strength and the margin to the reception threshold are measured at each testing point and the results are given in Table V. Among the chosen points, the maximum distance from the receiver to the transmitter was about $39 \mathrm{~km}$, which proves the good coverage effect of DTMBA in the application of $4 \mathrm{~K}$ UHDTV thanks to its excellent performance.

\section{B. Field Trial Results of SFN in Hong Kong}

Hong Kong is characterized by concentrated high-rise

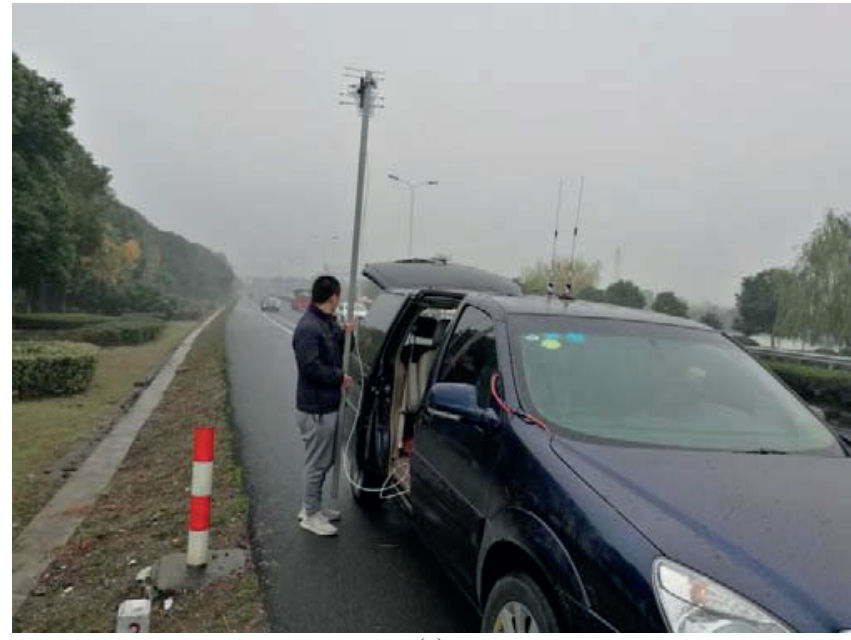

(a)

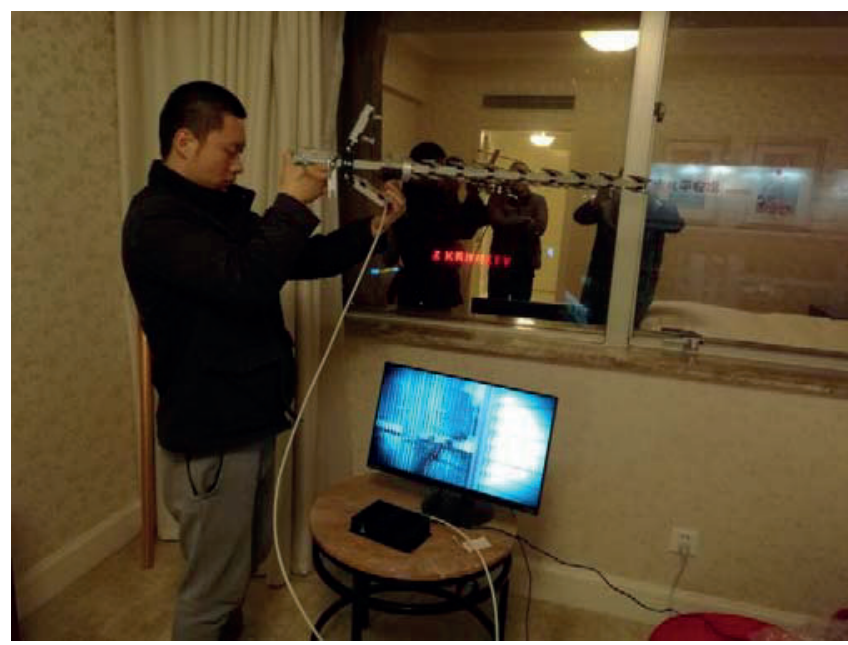

(b)

Fig. 12. The field trial in Jiaxing City. (a) Outdoor reception. (b) Indoor reception.

buildings and dense population, complex hilly urban terrain, and also unpredictable tidal effect, which gives a serious challenge to the coverage of the DTTB signal. Since 2007, Hong Kong has successfully established the SFN covering the whole area with good performance using DTMB.

In order to further prove the coverage performance of the DTMB-A system in Hong Kong, the SFN field trials were conducted in August 2019 using three DTMB-A transmitting stations to form the SFN [13]. Outdoor fixed reception, mobile reception, and the tidal fading tests were included for the measurements. One $4 \mathrm{~K}$ UHDTV program (data rate of $25.5 \mathrm{Mbps}$ ) and one $2 \mathrm{~K}$ HDTV program (data rate of $6 \mathrm{Mbps}$ ) using H.265 were selected for the fixed and mobile receptions, respectively.

Three transmitting stations of the tested SFN were located at Temple Hill, Golden Hill and Kowloon Peak, and their basic technical parameters are listed in Table VI. Three typical working modes were chosen, one for fixed reception and two for mobile reception. The detailed transmission parameters are 


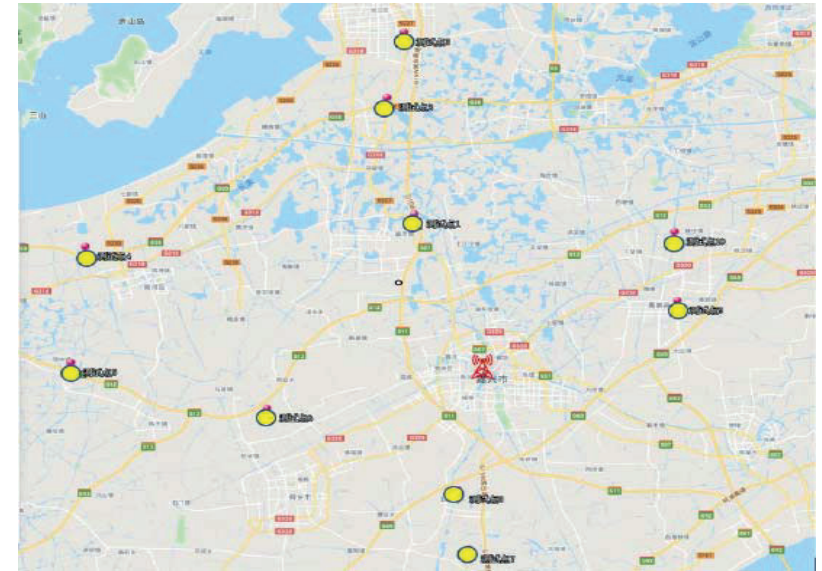

(a)

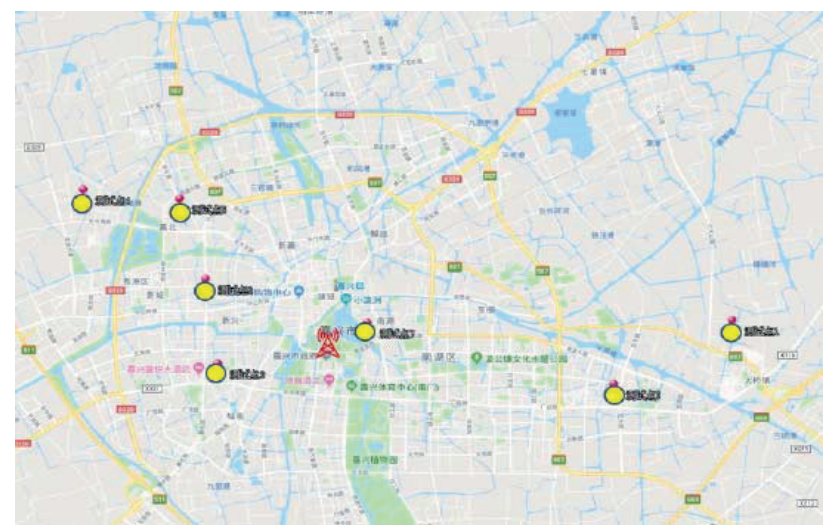

(b)

Fig. 13. The locations of the test points. (a) Outdoor reception. (b) Indoor reception.

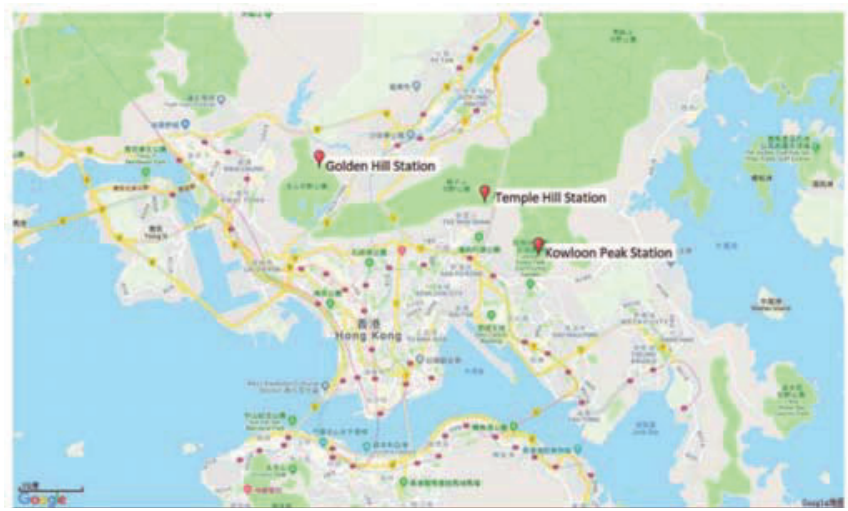

Fig. 14. The locations of the transmission stations in Hong Kong.

shown in Table VII.

The failure criterion of subjective evaluation was adopted in the fixed reception test. A failure according to the threshold of visibility (TOV) criterion was counted if three times of mosaic was observed within one minute. For the mobile reception, the DTMB-A receiver would give an error package indication signal from the LDPC decoder to a dedicated mobile test recorder. The recorder would record the time, the coordinates

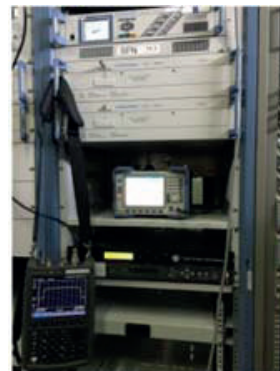

Temple Hill Station
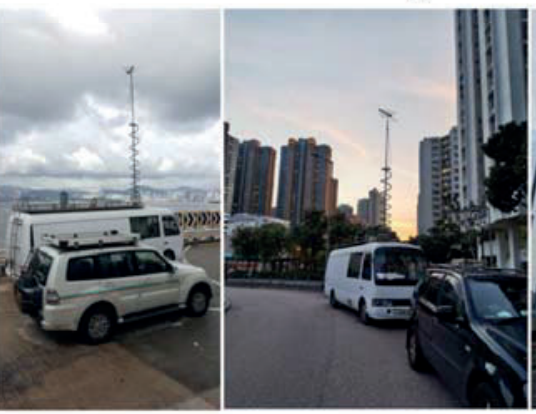

(b)

Fig. 15. The field trial conditions in Hong Kong. (a) Transmission stations. (b) Survey Car.

TABLE VI

TRANSMITTER PARAMETERS IN HONG KONG

\begin{tabular}{|c|c|c|c|}
\hline & Temple Hill & Golden Hill & Kowloon Peak \\
\hline ERP & $1000 \mathrm{~W}$ & $320 \mathrm{~W}$ & $320 \mathrm{~W}$ \\
\hline Frequency & \multicolumn{3}{|c|}{$602 \mathrm{MHz}$} \\
\hline Antenna type & \multicolumn{3}{|c|}{4 dipoles on the reflector } \\
\hline Polarization & \multicolumn{3}{|c|}{ Horizontal polarization } \\
\hline Antenna height & $527 \mathrm{~m}$ & $404 \mathrm{~m}$ & $651 \mathrm{~m}$ \\
\hline Location & $\begin{array}{l}22.35595^{\circ} \mathrm{N} \\
114.20665^{\circ} \mathrm{E}\end{array}$ & $\begin{array}{l}22.36258^{\circ} \mathrm{N} \\
114.14534^{\circ} \mathrm{E}\end{array}$ & $\begin{array}{l}22.34089^{\circ} \mathrm{N} \\
114.22333^{\circ} \mathrm{E}\end{array}$ \\
\hline
\end{tabular}

TABLE VII

PARAMETERS OF THE FIELD TRIALS IN HONG KONG

\begin{tabular}{|l|c|c|c|}
\hline \multicolumn{1}{|c|}{ Parameter } & Fixed & Mobile1 & Mobile2 \\
\hline Bandwidth & \multicolumn{3}{|c|}{$7.56 \mathrm{MHz}$} \\
\hline Constellation & $256 \mathrm{APSK}$ & 16APSK & $64 \mathrm{APSK}$ \\
\hline FFT size & $32 \mathrm{~K}$ & $4 \mathrm{~K}$ & $4 \mathrm{~K}$ \\
\hline Guard interval & \multicolumn{3}{|c|}{ Dual PN-MC 1024 } \\
\hline LDPC length & \multicolumn{3}{|c|}{61440} \\
\hline LDPC rate & $2 / 3$ & $1 / 2$ & $1 / 2$ \\
\hline Payload data rate & $37.89 \mathrm{Mbps}$ & 10.07 & 15.10 \\
\hline
\end{tabular}

of the location, and the corresponding field strength of the received signal when the error package appeared.

In order to establish DTMB-A SFN, the SFN adapter and the TS player were equipped in Temple Hill station besides DTMBA transmitter. The generated TS from the TS player is processed by the SFN adapter and a special packet carrying SFN synchronization information is inserted into the TS signal. The output TS is transmitted to Golden Hill and Kowloon Peak stations through the program distribution link, and radiated by the DTMB-A transmitters. In order to guarantee the clock 


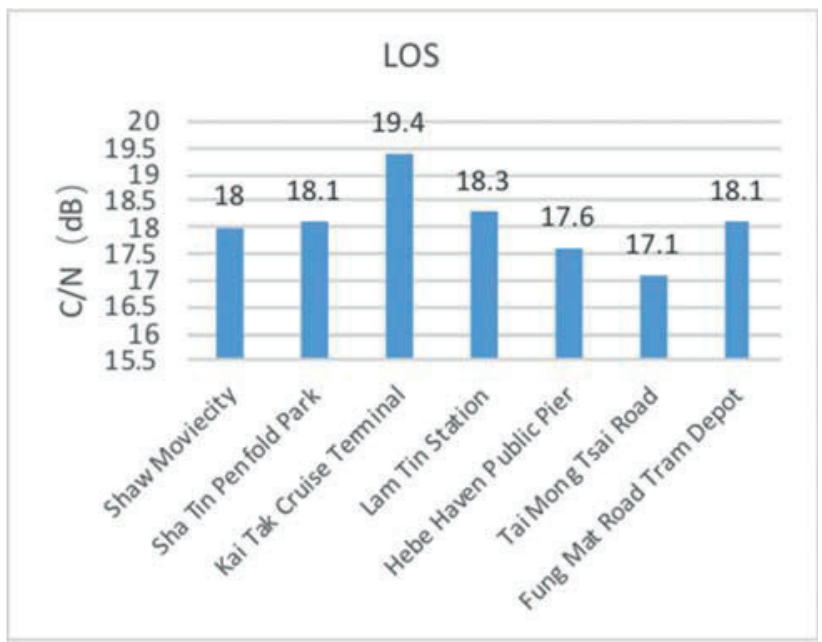

Fig. 16. Measurement results of fixed reception under LOS

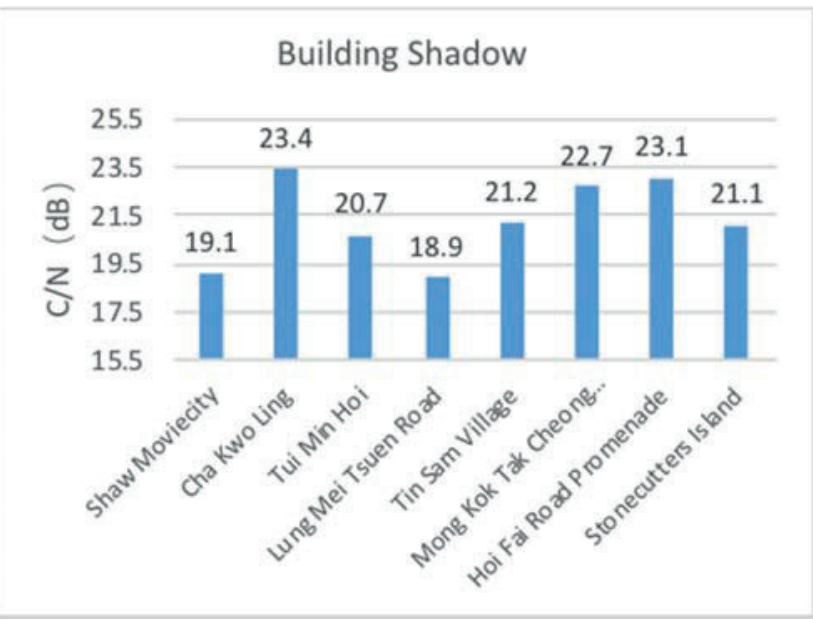

Fig. 17. Measurement results of fixed reception under building shadow.

synchronization of all stations, a global position system (GPS) timing receiver is set up at each station to provide stable clock reference for all equipment. The geographical location of the three stations is shown in Fig. 14. The photos of the equipment construction at the stations and the situations of the field trials are presented in Fig. 15.

\section{1) Fixed Reception}

The performance of the fixed reception is meaningful to check the coverage effect of the DTMB-A signal in Hong Kong. Thus, a large number of fixed reception test points were selected to evaluate the receiving quality. The $\mathrm{C} / \mathrm{N}$ threshold and the minimum reception level under line-of-sight (LOS) and building shadow conditions were mainly measured in the test.

Figs. 16 and 17 give the $\mathrm{C} / \mathrm{N}$ of various testing points under LOS and building shadow conditions, respectively. The figures indicate that DTMB-A with $8 \mathrm{MHz}$ bandwidth can provide reliable signal reception and support $4 \mathrm{~K}$ UHDTV under the above mentioned two conditions.

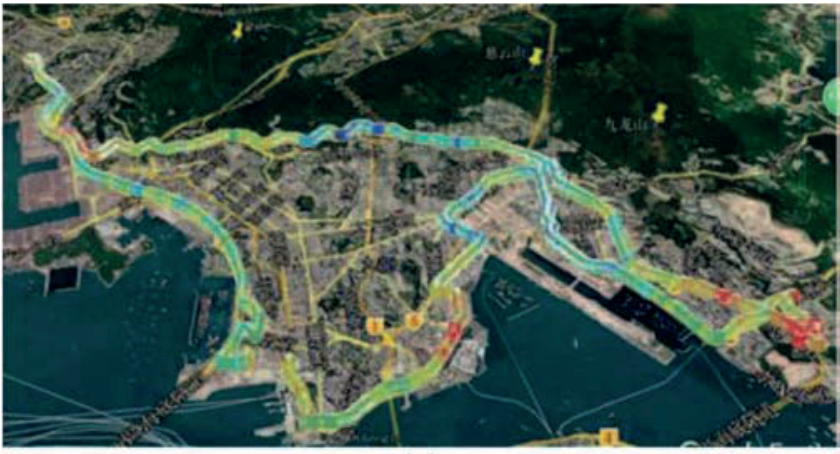

(a)

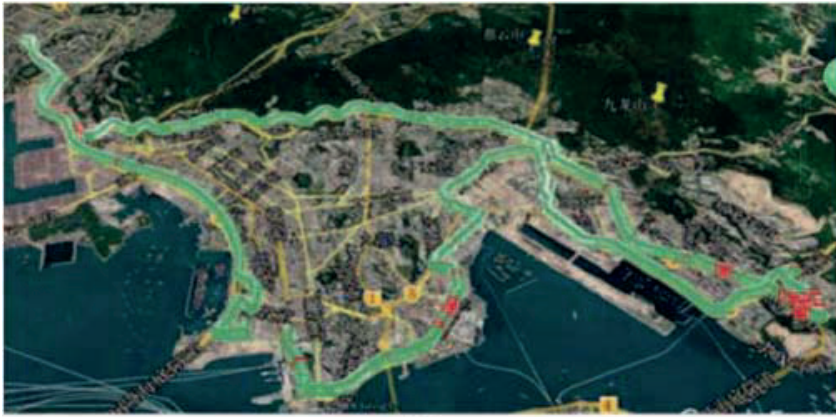

(b)

Fig. 18. Measurement results for mobile reception. (a) Signal Strength. (b) Block error

\section{2) Mobile Reception}

Mobile reception is important to mobile terminals such as vehicle TVs and smartphones. Thus, the performance of mobile reception was fully tested on typical routes including Hong Kong Island Expressway, Kowloon Island loop line and Nathan Road.

Fig. 18 shows the results of mobile reception tests on the Kowloon Island loop line. Fig. 18 (a) gives the received signal strengths recorded at one-second intervals over the range from $-90 \mathrm{dBm}$ to $-55 \mathrm{dBm}$. Different colors indicate different signal strengths, for example, the colors from red to yellow, green and blue mean the signal power changing from weak to strong. Fig. 18 (b) gives the block error results of the same points as (a), where green denotes error-free and red means the occurrence of decoding error. It is clear that except for the positions where the received signal strength was about or lower than $-90 \mathrm{dBm}$, the DTMB-A receiver could recover the video programs without error on most parts of the test route.

The selected routes of the whole test referred to the field trials of DTMB. The test results fully prove that DTMB-A can achieve the same coverage effect as DTMB with over $30 \%$ higher spectrum efficiency. In other words, DTMB-A can provide better performance or larger coverage area under the same spectrum efficiency as DTMB.

\section{CONCLUSION}

DTMB and DTMB-A both adopt TDS-OFDM as their basic physical layer transmission scheme. Compared to DTMB, DTMB-A can provide higher spectrum efficiency, better 
receiving reliability, and stronger robustness to the multipath. Thus, DTMB-A can support more applications with better service quality. This paper focuses on the details of the physical layer transmission techniques of DTMB and DTMB-A, showing the similarities and differences, and presents field trial results of the DTMB-A system. The results confirm that DTMB-A can provide reliable signal reception under various typical complicated receiving conditions, reliable coverage for both fixed and mobile reception. DTMB-A based SFN works well and 4K UHDTV programs can be transmitted through a DTMB-A network successfully.

\section{REFERENCES}

[1] ATSC Digital Television Standard, ATSC Standard, A/53 parts 1-6, Jan. 2007.

[2] Digital Video Broadcasting (DVB); Framing Structure, Channel Coding and Modulation for Digital Terrestrial Television, ETSI Standard EN 300 744 v1.6.2, Oct. 2015

[3] Transmission System for Digital Terrestrial Television Broadcasting, ARIB Standard STD-B31 Version 2.2, Mar. 2014

[4] Framing Structure, Channel Coding and Modulation for Digital Television Terrestrial Broadcasting System, Chinese National Standard GB20600-2006, Aug. 2006

[5] J. Song et al., "Technical review on Chinese digital terrestrial television broadcasting standard and measurements on some working modes," IEEE Trans. Broadcast., vol. 53, no. 1, pp. 1-7, Mar. 2007.

[6] J. Song, Z. Yang, and J. Wang, Digital Terrestrial Television Broadcasting: Technology and System. Piscataway, NJ, USA: Wiley, 2015.

[7] Digital Video Broadcasting (DVB); Frame Structure Channel Coding and Modulation for a Second Generation Digital Terrestrial Television Broadcasting System (DVB-T2), ETSI EN 302755 v1.4.1, Jul. 2015.

[8] ATSC Standard: Physical Layer Protocol (A/322), Adv. Television Syst. Committee, Washington, DC, USA, Jun. 2017.

[9] J. Song and C. Zhang, "Technical review on DTMB-advanced (DTMBA) standard," in Proc. Int. Conf. Eng. Telecommun. (EnT), Nov. 2016, pp. $128-133$.

[10] J. Song, C. Zhang, K. Peng, J. Wang, C. Pan, F. Yang, J. Wang, H. Yang, Y. Xue, Y. Zhang and Z. Yang, "Key technologies and measurements for DTMB-A system," IEEE Trans. Broadcast., vol. 65, no. 1, pp. 53-64, Mar. 2019.

[11] Error-correction, data framing, modulation and emission methods and selection guidance for second generation digital terrestrial television broadcasting systems, Recommendation ITU-R BT.1877-2, Dec. 2019.

[12] C. Pan, J. Wang, H. Fang and J. Song, " Field trial of advanced DTMB system DTMB-A in Hong Kong," in Proc. Int. Symp. Broadband Multimedia Syst. Broadcast. (BMSB), pp. 1-4, Jun. 2013.

[13] C. Pan, C. Zhang, H. Yang, J. Wang and X. Li, "Results of the DTMBA field trials in Hong Kong," in Proc.Int. Conf. Engineering and Telecommunication (EnT), Nov. 2019

[14] Y. Xue, H. Yang, C. Pan and J. Song, "Field trial of UHDTV over digital television terrestrial broadcasting network," in Proc. Int. Symp. Broadband Multimedia Syst. Broadcast. (BMSB), Jun. 2019.

[15] K.-J. Kim et al., "Low-density parity-check codes for ATSC 3.0," IEEE Trans. Broadcast., vol. 62, no. 1, pp. 189-196, Mar. 2016.

[16] A. I. V. Casado, W.-Y. Weng, S. Valle, and R. D. Wesel, "Multiple-rate low-density parity-check codes with constant blocklength," IEEE Trans. Commun., vol. 57, no. 1, pp. 75-83, Jan. 2009.

[17]Z. Liu, Q. Xie, K. Peng, and Z. Yang, "APSK constellation with gray mapping," IEEE Commun. Lett., vol. 15, no. 12, pp. 1271-1273, Dec. 2011.

[18] X. Li and J. A. Ritcey, "Bit-interleaved coded modulation with iterative decoding," IEEE Commun. Lett., vol. 1, no. 6, pp. 169-171, Nov. 1997.

[19]T. Cheng, K. Peng, Z. Liu, and Z. Yang, "Efficient receiver architecture for LDPC coded BICM-ID system," IEEE Commun. Lett., vol. 19, no. 7, pp. 1089-1092, Jul. 2015.

20]Z. Yang, X. Wang, Z. Wang, J. Wang, and J. Wang, "Improved channel estimation for TDS-OFDM based on flexible frequency-binary padding," IEEE Trans. Broadcast., vol. 56, no. 3, pp. 418-424, Sep. 2010

[21] J. Fu, J. Wang, J. Song, C.-Y. Pan, and Z.-X. Yang, "A simplified equalization method for dual PN-sequence padding TDS-OFDM systems," IEEE Trans. Broadcast., vol. 54, no. 4, pp. 825-830, Dec. 2008
[22]Z. Gao, C. Zhang, and Z. Wang, "Robust preamble design for synchronization, signaling transmission, and channel estimation," IEEE Trans. Broadcast., vol. 61, no. 1, pp. 98-104, Mar. 2015.

[23] J. Liu, C. Zhang and C. Pan, "Reliable transmission parameter signaling detection for DTMB-A standard," IEICE Trans. Commun., vol. E100-B, no. 12, pp. 2156-2163, Dec. 2017.

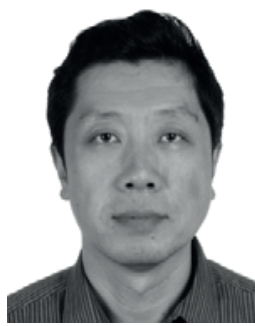

Jian Song received all his degrees in electrical engineering from Tsinghua University, Beijing China. He was with the Chinese University of Hong Kong, Hong Kong and University of Waterloo, Canada, in 1996 and 1997, respectively. He worked for industry in the US for seven years before joining the faculty team at Tsinghua University as a Professor in 2005. He is currently the Director of the Tsinghua's DTV Technology Research and Development Center. His research interests include digital television terrestrial broadcasting, wireless communication, power line communication, and visible light communication, and he is the recipient of the IEEE Scott Helt Memorial Award in 2015. He has published over 240 peerreviewed journal and conference papers in the aforementioned areas and one book in DTV area by Wiley, holds two U.S. and over 40 Chinese patents. He is a Fellow of IEEE, IET and CIE.

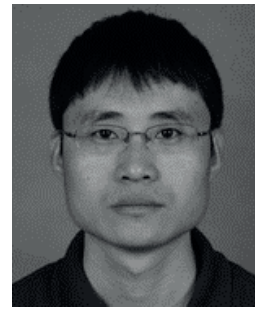

Chao Zhang received the B.S. and Ph.D. degrees from Beihang University in 2001 and 2008, respectively. From 2008 to 2010, he was a PostDoctoral Fellow with the Department of Electronic Engineering, Tsinghua University, Beijing, China. From 2011, he was an Assistant Professor with the Department of Electronic Engineering, Tsinghua University. He is currently an Associate Professor with the Department of Electronic Engineering, Tsinghua University. He has authored over 50 journal and conference papers. He holds over 20 Chinese patents. His research interests are in wireless and visible light communications with an emphasis on OFDM, MIMO, synchronization, channel estimation and channel equalization. He received the IEEE Scott Helt Memorial Award (Best Paper Award in IEEE TRANSACTIONS ON BROADCASTING) in 2016.

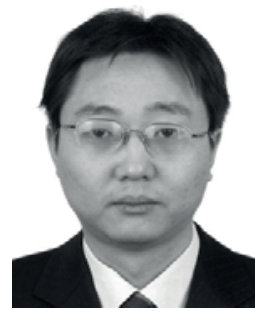

Jintao Wang received the B.Eng. and Ph.D. degrees in electrical engineering both from Tsinghua University, Beijing, China, in 2001 and 2006, respectively. From 2006 to 2009, he was an Assistant Professor in the Department of Electronic Engineering at Tsinghua University. Since 2009, he has been an Associate Professor and Ph.D. Supervisor. $\mathrm{He}$ is a [24] Standard Committee Member for the Chinese national digital terrestrial television broadcasting standard. His current research interests include space-time coding, MIMO, OFDM, and SFN systems. He has published more than 100 journal and conference papers and holds more than 40 national invention patents.

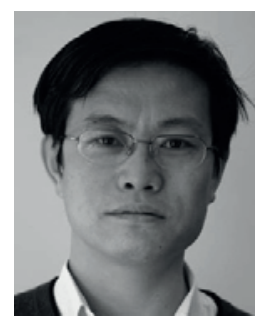

Yonglin Xue got his Bachelor and Master degrees from the Department of Electronic Engineering, Tsinghua University and Chinese Academy of Sciences in 1989 and 1992, respectively. He is now an Associate Professor with the Research Institute of Information Technology of Tsinghua University with major research interest in the digital TV broadcasting, video coding and communication. He has published more than 70 academic papers. 


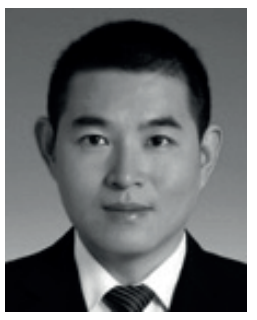

Changyong Pan, born in Anhui province, China, in 1975 , is a full professor in the Research Institute of Information Technology and deputy director of DTV R\&D Center of Tsinghua University. Prof. Pan has authored or co-authored more than 180 technica papers and published 8 technical books. He holds 34 patents, won national technical awards three times and is also the winner of numerous other awards

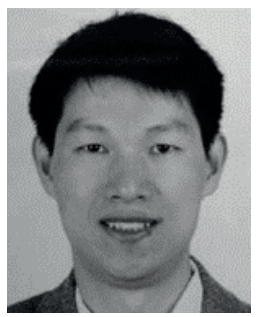

Kewu Peng was born in Hefei, China. He received the B. Eng degree in Electronics Engineering from Hefei University of Technology in 1993, the M.E. degree in Electronics Engineering from Tsinghua University in 1996, and the Ph.D. degree in Electrical Engineering from the University of Minnesota, Minneapolis, in 2003. He was a Researcher and a Lecturer with the Department of Electronic Engineering, Tsinghua University, from Aug 1996 to Aug 1999. Since Jan 2005, he joins the Digita Television Research Center at Tsinghua University as a Research Staff, Assistant Researcher('06), and Associate Professor('09). His research interests include mobile/wireless communications, digital terrestrial/television broadcasting, and image/video transmission. Currently, he is working on LDPC-coded non-orthogonal multiple access. Dr. Peng has published more than 90 journal and conference papers and holds more than 60 China patents.

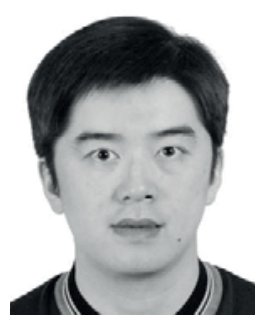

Yang Fang received the B.S.E. and Ph.D. degrees in electronic engineering from Tsinghua University, Beijing, China, in 2005 and 2009, respectively. He is currently an Associate Professor with the Research Institute of Information Technology, Tsinghua University. He has authored over 120 peer-reviewed journal and conference papers. He holds over 40 Chinese patents and two PCT patents. His research interests lie in the fields of channel coding, channel estimation, interference cancelation, and signal processing techniques for communication systems, especially in power line communication, visible light communication, and digital television terrestrial broadcasting. He received the IEEE Scott Helt Memorial Award (Best Paper Award in IEEE TRANSACTIONS ON BROADCASTING) in 2015. He is the Secretary General of Sub-Committee 25 of the China National Information Technology Standardization (SAC/TC28/SC25).

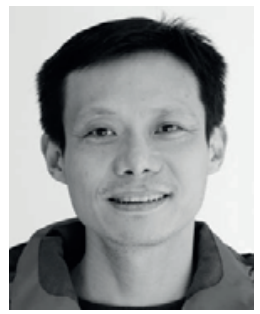

Jun Wang was born in Henan, China, on October 5 , 1975. He received the B.Eng. and Ph.D. degrees from the Department of Electronic Engineering at Tsinghua University, Beijing, China, in 1999 and 2003, respectively. He has been Assistant Professor and member of the DTV Technology R\&D Center of Tsinghua University since 2000 . His main research interests focus on broadband wireless transmission techniques, especially synchronization and channel estimation. He is actively involved in the Chinese national standard on the Digital Terrestrial Television Broadcasting technical activities, and has been selected by the Standardization Administration of China as the Standard committee member for drafting.

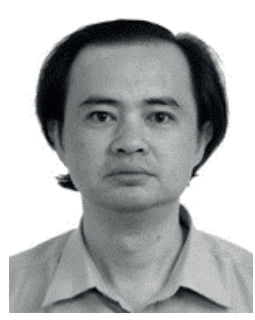

Hui Yang was born in Guangxi province, China, in 1967. He received his Master degree from the Department of Electronic Engineering, Tsinghua University. Now, he is the Senior Engineering with the Research Institute of Information Technology of Tsinghua University. His main research interests include digital television transmission, visible light communications and power-line communications, etc. He has published more than 100 technical papers and holds more than 20 patents.

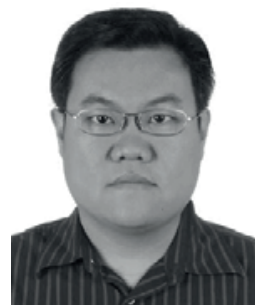

Yu Zhang received the B.E. and M.S. degrees in electronics engineering both from Tsinghua University, Beijing, China, in 1999 and 2002, respectively, and the $\mathrm{Ph} . \mathrm{D}$. degree in electrical and computer engineering from the Oregon State University, Corvallis, OR, USA, in 2006. From 2007, he was an Assistant Professor with the Research Institute of Information Technology, Tsinghua University, for eight months. He is currently an Associate Professor with the Department of Electronic Engineering, Tsinghua University. His current research interests include the performance analysis and detection schemes for MIMO-OFDM systems over doubly-selective fading channels, transmitter and receiver diversity techniques, and channel estimation and equalization algorithm.

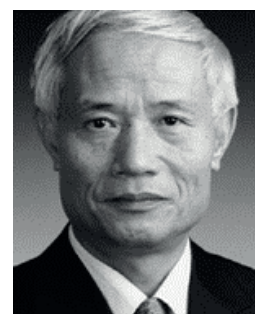

Zhixing Yang, born in Hunan China in 1946, is a full professor and $\mathrm{PhD}$ advisor with the Department of Electronic Engineering, Tsinghua University, Beijing, China. He is the deputy director of the State Key Lab on Microwave and Digital Communications, Director of the Digital TV (DTV) transmission technology $\mathrm{R} \& \mathrm{D}$ center at Tsinghua University. He is also a member of the special committee of National DTV standardization, group leader of the coordinating working group for DTV standardization of MII, the invited committee member of the science and technology committee of SARFT. He is now the chairman of the National Engineering Lab. for DTV (Beijing).

He has been focusing on the various transmission technologies for both telecommunications and broadcasting areas. Prof. Yang has won national technical invention awards three times and is also the winner of numerous other awards. Prof. Yang is the first draftsman of the Chinese Digital Television Terrestrial Broadcasting Standard, DTMB (GB20600-2006). 